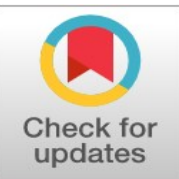

\title{
A hybrid control design of FOPID and FWA for inverted pendulum systems
}

\author{
Te-Jen Su ${ }^{{ }^{*}}$, Tung-Yeh Tsou ${ }^{2}$, Shih-Mine Wang ${ }^{3}$, Van-Manh Hoang ${ }^{4}$, Kuo-Win Pin ${ }^{5}$ \\ 1, 2, 3, 4, 5 National Kaohsiung University of Applied Sciences, Kaohsiung, Taiwan
}

\author{
Index Terms \\ FWA \\ FOPID \\ Sliding Mode Control \\ Fast Output Sampling \\ Inverted Pendulum \\ Received: 2 July 2016 \\ Accepted: 5 August 2016 \\ Published: 25 October 2016
}

\begin{abstract}
Nowadays, along with the dramatic development of industrial automatic, optimization problem has been playing an important role in designing controllers for nonlinear systems. This paper proposes a hybrid control design of Fast Output Sampling Discrete Sliding Mode Control (FOSDSMC) and fractional order PID controller (FOPID) based on fireworks algorithm (FWA) to optimize controller parameters. The hybrid controller is verified on a nonlinear inverted pendulum system. The simulation of controller optimization process is carried out using MATLAB/Simulink. The results are compared with two published controllers such as a hybrid control design of PID controller and fast output sampling discrete sliding mode control, and a hybrid control configuration of PID and state feedback controller based on linear quadratic regulator method. The comparison results show the better performance of the proposed method.
\end{abstract}

\section{INTRODUCTION}

The inverted pendulum system is known as one of the most popular benchmark objects in verifying the validity of control techniques. The system consists of 2 main parts: pendulum and cart. The aim in designing its controller is to move the cart to desired position while still keeping the pendulum balanced in vertical axis. The system has two outputs: position of the cart and angle of the pendulum; the input is driving force applied to cart. Because the system has a high nonlinearity, using pure linear controllers as PID has been becoming a very hard task. By this way, more nonlinear controllers have been arising with the aim of controlling this system.

Some common approaches can be known as sliding mode controllers (SMC) $1 \sim 3$, model predictive controller [4], fuzzy logic controller [5], fuzzy-neural controller [6], and so on. The performance of SMC controller has been improved by applying good techniques such as fast output sampling (FOS) technique $[1,3]$. A hybrid approach of PID and FOS feedback discrete SMC is developed [2]. So far a fractional order PID (FOPID) [7] is more preferred than a

\footnotetext{
${ }^{*}$ Corresponding author: Te-Jen Su

${ }^{\dagger}$ Email: sutj@kuas.edu.tw
}

classical PID controller in a wide area of the controlling linear and nonlinear systems.

In fact, the dominant performance of FOPID is verified to increase quality and robustness of the controller [8]. Actually, there are many methods to determine the FOPID parameters based on synthesizing coefficients using performance criterion as ITAE [7]. Tuning FOPID controller parameters based on these methods often not only takes much time to choose an acceptable solution, but the accuracy is also not high. So far arising evolutionary algorithm such as genetic algorithm (GA) [9], particle swarm optimization (PSO) [10], and so on can make this work become easier. However, searching process of the heuristic algorithms such as GA often finds local optima that sometimes are far away from the global optima; especially the convergence speed of GA is so slow that takes many generations to obtain the optima.

In recent years, Fireworks Algorithm (FWA) is developed as evolutionary algorithm. The main idea of the FWA is to use the explosion of the fireworks to search the feasible space of optimization function. It has noted that FWA has higher optimization accuracy as well as faster conver- 
gence speed as compared with PSO [11]. Therefore, this paper proposes a hybrid control design of FOSDSMC which has an advantage of making both position and angle variable approach to equivalent point, and FOPID which adjusts the cart tracking desired positions. The FWA will adjust FOPID controller parameters to improve performance of the nonlinear inverted pendulum system. This paper is organized as follows: Section 2 shows mathematic modeling of the inverted pendulum system. Section 3 briefly describes the methodology. Simulation results and comparison with other published controllers are demonstrated in Section 4. The conclusions are given in Section 5.

\section{MODELING OF THE INVERTED PENDULUM SYSTEM}

The inverted pendulum system is shown in Figure 1. The cart is driven by an external force that drives a pair of wheels of the cart. Each wheel of the cart is driven by a DC motor.

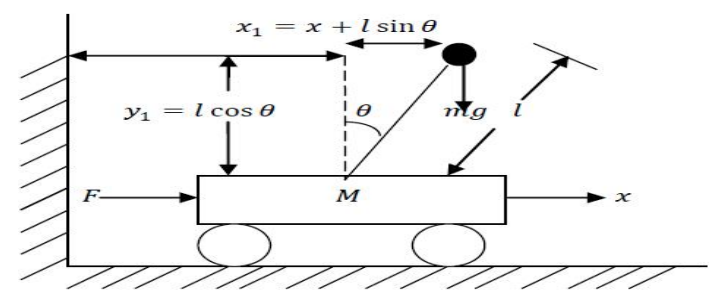

Fig. 1. The inverted pendulum system

Consider the coordinate system Oxy whose origin is at the center of the cart with the equilibrium point. As shown in Figure 1, $\theta$ is the angle of the inverted pendulum from the vertical axis, $\mathrm{x}$ is the displacement of the cart, and $\mathrm{F}$ is driving force applied to the system. $\mathrm{M}$ is the mass of the cart; $m$ is the ball point mass as the upper end of the inverted pendulum (mass of the rod is negligible); $l$ is the length of the pendulum rod. Assume that the inertia moment of pendulum and frictional force are negligible. The system dynamics are described as follows:

$$
\left\{\begin{array}{l}
\ddot{x}=\frac{F+m l \sin (\theta) \dot{\theta}^{2}-m g \sin \theta \cos \theta}{M+m-m \cos ^{2} \theta} \\
\ddot{\theta}=\frac{F \cos \theta-(M+m) g \sin \theta+m l(\sin \theta+\cos \theta) \dot{\theta}^{2}}{m l \cos ^{2} \theta-(M+m) l}
\end{array}\right.
$$

The state variables are defined as position, velocity of the cart, angle, and angular velocity of the pendulum, respectively:
Then, the above dynamic equations can be represented in the form of the state space equations as below:

$$
\left\{\begin{array}{l}
\dot{x}_{1}=x_{2} \\
\dot{x}_{2}=\frac{F+m l \sin (\theta) \dot{\theta}^{2}-m g \sin \theta \cos \theta}{M+m-m \cos ^{2} \theta} \\
\dot{x}_{3}=x_{4} \\
\dot{x}_{4}=\frac{F \cos \theta-(M+m) g \sin \theta+m l(\sin \theta+\cos \theta) \dot{\theta}^{2}}{m l \cos ^{2} \theta-(M+m) l}
\end{array} .\right.
$$

The output equation can be written as:

$$
y=\left[\begin{array}{llll}
1 & 0 & 0 & 0 \\
0 & 0 & 1 & 0
\end{array}\right]\left[\begin{array}{c}
x \\
\dot{x} \\
\theta \\
\dot{\theta}
\end{array}\right]
$$

Fractional calculus is a generation of integration and differentiation to non-linear order fundamental operator, where $a$ and $t$ are the limits of the operation and. The continuous Integra-differential operator is defined as [12]:

$$
{ }_{a} D_{t}^{r}=\left\{\begin{array}{ccc}
\frac{d^{r}}{d t^{r}} & \text { if } & r>0 \\
1 & \text { if } & r=0 \\
\int_{a}^{t}(d \tau)^{-r} & \text { if } & r<0
\end{array}\right.
$$

There are some definitions on fractional-order differentiations, such as Riemann-Liouville's definition, definition, Caputo's definition, and others. From numerical implementation point of view, Grünwald-Letnikov's definition that is one of the most common definitions is given by the following formula [7]:

$$
D_{t}^{\prime} \approx \frac{1}{h^{\alpha}} \sum_{j=0}^{t-a / h} w_{j}^{(\alpha)} f(t-j h)
$$

where the binomial coefficients can recursively be calculated by the following formula: 


$$
\left\{\begin{array}{l}
w_{0}{ }^{(\alpha)}=1 \\
w_{j}{ }^{(\alpha)}=\left(1-\frac{\alpha+1}{j}\right) w_{j-1}^{(\alpha)} \quad(j=1,2, \ldots)
\end{array}\right.
$$

\section{IMPLEMENTATIONS OF FRACTIONAL-ORDER PID}

\section{CONTROLLER}

The most common form of a fractional order PID controller is the controller, where an integrator order and a differentiator order can be any real numbers. The transfer function of a fractional order controller has the form:

$$
G_{C}(s)=K_{P}+\frac{K_{I}}{s^{\lambda}}+K_{D} s^{\mu}, \quad \lambda, \mu>0
$$

The PID , PI , and PD controllers are three special cases of controller. There are two common implementations or approximations of fractional - order operators and system, such as an Oustaloup's approximation and a modified Oustaloup's approximation. When fractional - order controllers have to be implemented or simulations have to be performed, fractional-order transfer functions are usually replaced by integer-order transfer functions with a behavior close enough to that desired, but much easier to handle. An Oustaloup's recursive approximation to fractional-order operators is good enough in most cases. This paper focuses on this approximation that is shown as follows [7]:

$$
G_{f}(s)=K \prod_{k=1}^{N} \frac{s+w_{k}^{\prime}}{s+w_{k}}
$$

And the poles, zeros, and gain are evaluated from:

$$
\left\{\begin{array}{l}
\omega_{k}^{\prime}=\omega_{b} \omega_{u}^{(2 k-1-\gamma) / N} \\
\omega_{k}=\omega_{b} \omega_{u}^{(2 k-1+\gamma) / N} \\
K=\omega_{h}^{\gamma} \\
\omega_{u}=\sqrt{\omega_{h} / \omega_{b}}
\end{array}\right.
$$

where:

$\mathrm{N}$ : Integer order of filter

$\gamma$ : Integer order of differentiator

ISSN: 2414-3103

DOI: $10.20474 /$ japs-2.3.4 $[b, h]$ : Pre-specified frequency range

\section{A. Fast Output Sampling Discrete Sliding Mode Controller}

For discrete sliding mode, control signal is activated at sampling instant and it is held constant during each sampling interval. In designing this controller, it often requires measuring all system states that sometimes is not feasible. To solve this problem, the fast output sampling feedback is applied to discrete sliding mode control. This technique is used to sample the output of system at a faster rate as compared to control input. The fast output sampling discrete sliding mode controller (FOSDSMC) can guarantee the stability of the closed loop system. The designing FOSDSMC controller with control law $\mathrm{u}_{-} \mathrm{k}$ and sliding surface function s_k are shown as follows [2]:

$$
\begin{aligned}
& u_{k}=F_{S} L_{y} y_{k}+F_{S} L_{u} u_{k-1} \\
& s_{k}=c^{T} L_{y} y_{k}+c^{T} L_{u} u_{k-1}
\end{aligned}
$$

\section{B. Fireworks Algorithm}

The natural processes based algorithms such as GA, PSO are often good choices when the cost function has only a few variables as well as narrow searching space. Otherwise, when the number of variables increases and the search space is larger, the computational time of these algorithms is not too much different. In this paper, FWA is proposed to optimize FOPID controller parameters. The advantages of FWA as compared with PSO, GA are high optimization accuracy and fast convergence speed. Because of spark generation and specific selection processes, the FWA can avoid premature convergence.

\section{FRAMEWORK}

Figure 2 shows the framework of FWA. Similar to explosion phenomenon of real fireworks, a shower of sparks will local space around a firework when it is set off. By this way, the explosion process of a firework can be viewed as a search in the local space around a specific point where the firework is set off through the sparks generated in the explosion. At the beginning of FWA, there are $\mathrm{n}$ fireworks which are set off at $\mathrm{n}$ given locations. Then after explosion, the locations of sparks are evaluated. When the optimal location is found, the algorithm stops. Otherwise, $n$ other locations are selected from the current sparks and current fireworks for the next generation of explosion. 


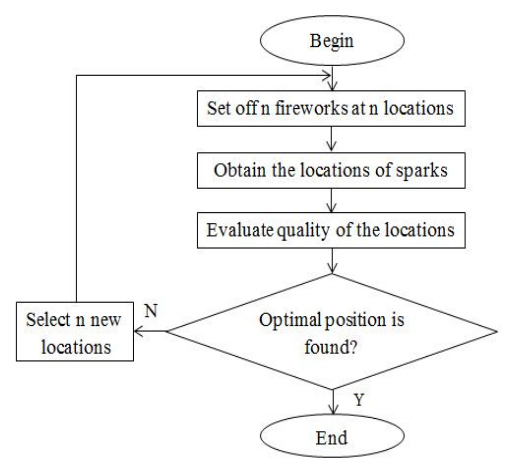

Fig. 2 . Framework of FWA program

There are two key factors to design a good firework such as number of sparks and explosion amplitude. A good firework should be satisfied that the number of sparks is as high as possible, while amplitude of explosion is as smallest as possible.

\section{A. Number of Spark}

Assume a general optimization problem as follows:

$$
\operatorname{Min} f(x) \in R, x_{\text {min }} \leq x \leq x_{\text {max }}
$$

where represents a location in the searching space, is a cost function, and are the bounds of the potential space. The number of sparks generated by each firework xi is shown as [11]:

$$
s_{i}=m \cdot \frac{y_{\max }-f\left(x_{i}\right)+\gamma}{\sum_{i-1}^{n}\left(y_{\max }-f\left(x_{i}\right)\right)+\gamma},
$$

where:

$\mathrm{m}$ : Maximum sparks

: The maximum value of object function, with

: The smallest constant to avoid zero division zero.

To avoid overwhelming effects of splendid fireworks, is defined in a range as below:

$$
\hat{s}_{i}=\left\{\begin{array}{cl}
\operatorname{round}(a . m) & \text { if } s_{i}<a . m \\
\operatorname{round}(b . m) & \text { if } s_{i}>b . m \\
\operatorname{round}\left(s_{i}\right) & \text { otherwise }
\end{array},\right.
$$

where $\mathrm{a}$ and $\mathrm{b}$ are constants,

\section{B. Amplitude of Explosion}

The amplitude of explosion is generated as the following formula [11]:

$$
A_{i}=\hat{A} \cdot \frac{f\left(x_{i}\right)-y_{\min }+\gamma}{\sum_{i=1}^{n}\left(f\left(x_{i}\right)-y_{\min }\right)+\gamma},
$$

where:

$\mathrm{A}^{\wedge}$ : The maximum explosion amplitude.

: The minimum value of the objective function:

\section{Cost Function}

In the optimal control problems, it often doesn't guarantee to find out exactly a global solution and the optimum value depends on the performance criteria which are used to formulate the cost function. In FOPID controller parameter optimizing problem, the cost function can be formulated from one or many different performance criteria. Now three typical performance criteria are given as follows: Integral of Time Multiplied by Square Error (ITSE):

$$
\text { ITSE }=\int t t^{2}(t) d t
$$

where $t$ denotes the current evaluation time, $e(t)$ is error value between set point and current output.

ITSE performance criterion may result in a response with a relative small overshoot as well as settling time, but it cannot guarantee to have a desirable stability margin.

\section{Mean Square Error (MSE):}

where $t$ denotes the current evaluation time, $e(t)$ is error value between set point and current output.

ITSE performance criterion may result in a response with a relative small overshoot as well as settling time, but it cannot guarantee to have a desirable stability margin.

\section{Mean Square Error (MSE):}

$$
\text { MSE }=\frac{1}{n} \sum_{i=1}^{n} e^{2}(t)
$$

where $\mathrm{n}$ is the length of simulation time.

MSE is effective to design lower order system. In the case of higher order systems, it makes the system response with higher values of settling time and percent of overshoot. 


\section{Integral of Square Time Multiplied by Square Error (ISTSE):}

$$
\text { ISTSE }=\int t^{2} e^{2}(t) d t
$$

The ISTSE, ITSE performance criteria can make the system response to overcome the disadvantages of Integral Of Absolute Value Of Error (IAE) and Integral Square Error (ISE). However, it has been stated [13] that it doesn't mean minimizing all the performance parameters of system response such as the percent of overshoot (P.O.), settling time (Ts), rising time (Tr), or steady state (Ess) at the same time. In multi-objective optimization problem, Pareto method which optimizes many different objectives at the same time is very popular. Nevertheless, when the number of objective functions increases, using this method becomes a hard task because of high complexity. In this paper the performance criteria are combined in a single weighted sum objective function that is defined as the following function:

$$
J=\sum_{i=1}^{n} w_{i} f_{i}(k)
$$

where:

f_i (k) : Performance criteria such as ISTSE, MSE, P.O., and so on. $\mathrm{n}$ : Number of performance criteria.

$\mathrm{w}_{-} \mathrm{i}$ : Weighted values of each performance criteria such that:

$$
\sum_{i=1}^{j} w_{i}=1
$$

\section{SIMULATION AND RESULTS}

Figure 3 and Figure 4 show the simulation model for the proposed optimization process and the hybrid control configuration block, respectively.

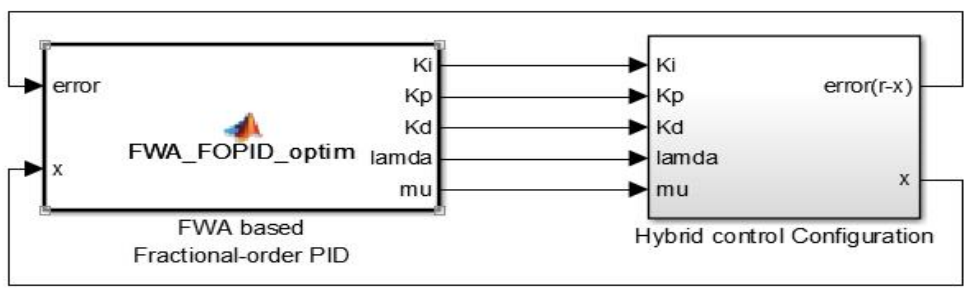

Fig. 3 . Simulation model for the proposed optimization process

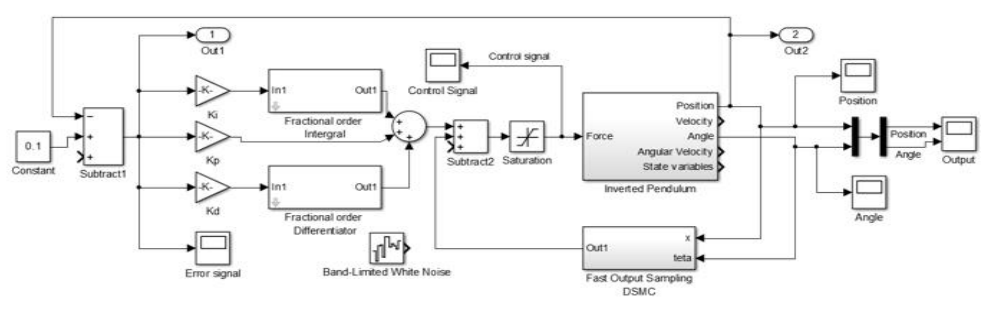

Fig. 4. The simulation model of the hybrid control configuration

The simulation parameters of inverted pendulum system are set as: the mass of the cart $2.4 \mathrm{~kg}$, mass of pendulum $0.23 \mathrm{~kg}$, length of pendulum $0.36 \mathrm{~m}$, gravity $9.8 \mathrm{~m} / \mathrm{s} 2$ and driving force is in range of [-20 20]N. In Figure 3, the "FWA_FOPID_optim" block is a function that is employed to optimize 5 parameters of the FOPID controller by using FWA. The proposed cost function of optimization process is formulated by ISTSE, MSE, ITSE and percent of overshoot parameter (PO).

$$
J=\alpha_{1} \cdot I S T S E+\alpha_{2} \cdot M S E+\alpha_{3} \cdot I T S E+\alpha_{4} \cdot P O
$$

The parameters of the cost function are set as: .The typical parameters of fireworks algorithm are set as: 5 optimized variables, including 3 PID parameters in range of [-100 100], order of two fractional order calculus blocks 
are in range of [0 2]; the number of fireworks $n=5$, the value of the total number of sparks $m=64$; the maximum explosion amplitude $A=2$; maximum iterations 300 ; maximum evaluation 50000; $a=0.04, b=0.8$. The controller parameters are designed at sampling time $0.05 \mathrm{~s}$. The fractionalorder derivative and differentiation block are designed as a mask block in Simulink based on the Oustaloup's filter. The frequency range of these two fractional calculus blocks is
[0.0001 1000]. The order of filter is 4 .

The dominant performance of the proposed controllers is verified by a comparison with two published controllers, including the hybrid controller of Reddy et al.'s and Prasad et al. Figure 5 and Figure 6 show response of position and angle in the cases of initial angle $0.1 \mathrm{rad}$, and zero initial conditions, respectively.

\section{RESULTS AND DISCUSSION}

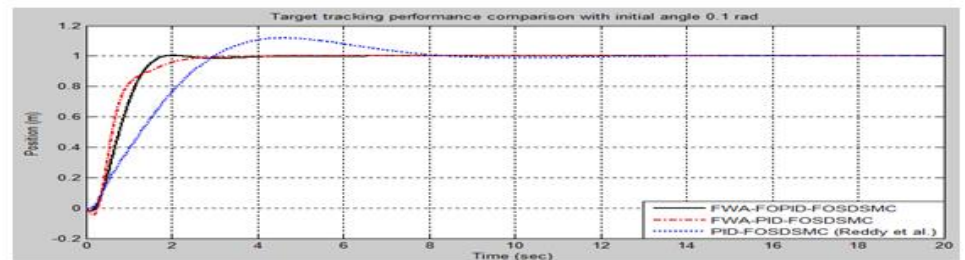

(a)

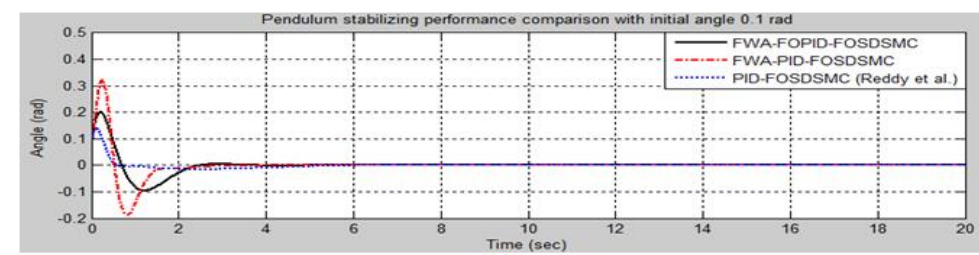

(b)

Fig. 5 . Response of (a) position (m); (b) angle (rad) with respect to time (sec) between the proposed methods and a published controller of [2]
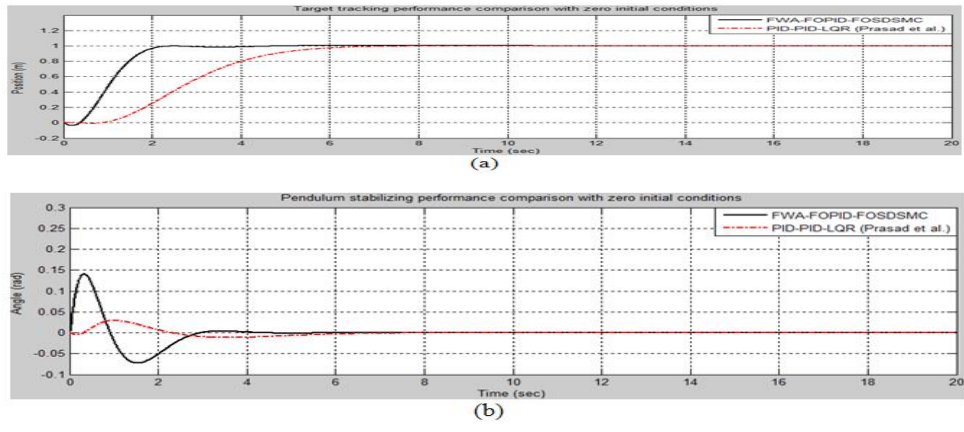

Fig. 6. Response of (a) position (m); (b) angle (rad) with respect to time (sec) between the proposed methods and a published controller of $[14]$

For inverted pendulum stabilizing, it is observed that the controllers can guarantee to stabilize the pendulum in the upright position very fast, even with different initial conditions of the system. For position tracking performance, in the case of determining FOPID or PID controller parameters is not good enough, it results in the system response with high overshoot as well as long settling time.
Instead, using fireworks algorithm to optimize FOPID controller parameters makes the system response much better that both the settling time and rising time are very small, while overshoot is approximate to zero. The responses of two proposed controllers, including FWA-FOPID-FOSDSMC and FWA-PID-FOSDSMC, are almost similar, but the response of proposed controller with FOPID is faster than that of PID controller. 


\section{CONCLUSION}

In this paper, the hybrid control design of fractionalorder PID and fast output sampling discrete sliding mode control based on FWA are proposed. Besides replacing classical PID by the fractional-order PID to combine with FOSDSMC, using FWA to optimize FOPID controller parameters shows a very good performance in the system response that not only guarantees stabilizing inverted pendulum balances at the upright position, but also highly enhances tracking performance. The simulation results show that the proposed controllers are much better than two published methods. As a result, the robust control of inverted pendulum system will be designed with the proposed method in the future.

\section{REFERENCES}

[1] C. C Kung, T. H. Chen and H. Y. Cheng, "Discrete sliding mode controller design with fast output sampling technique for discrete time T-S fuzzy system," in IEEE International Conference on Fuzzy Systems, Hong Kong, 2008, pp. 14191424.

[2] N. P. K. Reddy M. S. Kumar and D. S. Rao, "Control of nonlinear inverted pendulum system using PID and fast output sampling based discrete sliding mode controller," International Journal of Engineering Research and Technology, vol. 3, no. 10, pp. 1000-1006, 2014.

[3] M. C. Saaj, B. Bandyopadhyay and H. Unbehauen, "A new algorithm for discrete time sliding mode control using output sampling feedback," IEEE Transactions on Industrial Electronics, vol. 49, no. 3, pp. 518-523, 2002.

DOI: 10.1109 /TIE.2002.1005376

[4] C. Cheng Z. Zhao and H. Li, "MPC controller performance evaluation and tuning of single inverted pendulum device," Journal of Computers, vol. 8, no. 6, pp. 1560-1570, 2012.

[5] A. Jain D. Tayal and N. Sehgal, "Control of nonlinear inverted pendulum using fuzzy logic controller," Journal of Computer Applications, vol. 69, no. 27, pp. 7-11, 2013. DOI: 10.5120/12141-8278

[6] R. J. Wai and P. C. Chen, "Robust neural-fuzzy network control for robot manipulator including actuator dynamics," IEEE Transactions on Industrial Electronics, 53, no. 4, pp. 1328-1349, 2006. DOI: 10.1109/TIE.2006.878297

[7] C. A. Monje, Y. Chen, B. M. Vinagre, D. Xue and V. Feliu-Batlle, Fractional Order Systems and Controls: Fundamentals and Applications. New York, NY: Springer Verlag, 2010. DOI: 10.1007/978-1-84996-335-0

[8] Y. Q. Chen, "Ubiquitous fractional order controls?" in 2nd IFAC Workshop on Fractional Differentiation and its Applications, Porto, Portugal, 2006, pp. 481-492. DOI: 10.3182/20060719-3-pt-4902.00081

[9] M. R. Dastranj, N. Changizi and N. Khoori, "Design of optimal pid controller for inverted pendulum using genetic algorithm," Journal of Innovation, Management and Technology, vol. 3, no. 4, pp. 440-442, 2012.

DOI: $10.1007 / 978-3-642-13498-2$

[10] T. O. S. Hanafy, "Stabilization of inverted pendulum system using particle swarm optimization," in 8th International Conference on Informatics and Systems (INFOS), Cairo, 2012, pp. 207-210.

[11] Y. Tan and Y. C. Zhu, “Fireworks algorithm for optimization,” in Advances in Swarm Intelligent. Beijing, China: Springer Verlag, 2010, pp. 355-364.

[12] A. K. Mahmood and B. F. Mohammed, "Design optimal fractional order PID controller utilizing particle swarm optimization algorithm and discretization method," International Journal of Emerging Science and Engineering, vol. 1, no. 10, pp. 87-92, 2013.

[13] M. A. Sahib and B. S. Ahmed, "A new multi-objective performance criterion used in PID tuning optimization algorithms," Journal of Advanced Research, vol. 7, no. 1, 1-9, 2015.

[14] S. Prasad, R. T. Williams, A. K. Mahalanabis, L. H. Sibul, "A transform-based covariance differencing approach for some classes of parameter estimation problems," IEEE Transactions on Acoustics, Speech, and Signal Processing, vol. 36, no. 5, pp. 631-641, 1988. DOI: 10.1109/29.1573 